\title{
Sensitivity of American Option Prices with Respect to the Variations of Local Volatility
}

\author{
Nasir Rehman ${ }^{1, *}$, Sultan Hussain ${ }^{2}$ and Wasim Ul-Haq ${ }^{3}$ \\ ${ }^{1}$ Department of Mathematics and Statistics, Allama Iqbal Open University, Islamabad, Pakistan \\ ${ }^{2}$ COMSATS Institute of Information Technology, Abbotabad, Pakistan \\ ${ }^{3}$ Department of Mathematics, Abdul Wali Khan University, Mardan, Pakistan
}

Received: 25 Feb. 2013, Revised: 28 Apr. 2013, Accepted: 29 Apr. 2013

Published online: 1 Sep. 2013

\begin{abstract}
In mathematical finance there are two well known and traditional techniques to deal American options: Solving parabolic partial differential equations and using the probabilistic approach. In this paper, we use purely probabilistic approach. We consider standard one-dimensional diffusion model with local volatility that is a function of time and current stock price and where the risk-free interest rate is constant. We estimate the continuity of American option prices with respect to the corresponding local volatilities.
\end{abstract}

Keywords: American put option, stochastic differential equation, local volatility, stopping time.

\section{Introduction}

Unlike the contracts (Forwards, Futures), an option is the right (but not the obligation) to buy or sell an asset at a predetermined price and within a predetermined time. This predetermined price is called Strike price $(K)$ and the predetermined time is called Maturity time $(T)$. The price of the underlying asset at time $t$ is known as the spot price and is denoted by $\left(S_{t}\right), 0 \leq t \leq T$. Options are basically of two types: A call (put) option gives its holder the right to buy (sell) the underlying asset at a strike price $K$ within the maturity $T$. But if we look at exercise style the options are of many types. A European call (put) option gives its holder the right to buy (sell) the underlying asset at a strike price $K$ exactly at the maturity time $T$. While the writer of the corresponding European option has the obligation to sell or buy the underlying asset at strike price $K$ and maturity time $T$ if the holder chooses to exercise the option. On account of this risk, the writer receives money (premium) from the holder when it sells the option called the price of the option.

In contrast an American call (put) option gives its holder the right to buy (sell) the underlying at strike price $K$ at any time within the maturity. Clearly its price is higher than the European option because it includes the European option as a special case at $t=T$ and in addition early exercise opportunity.
We consider a probability space $(\Omega, \mathscr{F}, P)$ and $\left(W_{t}\right), 0 \leq t \leq T$, is a one-dimensional standard Brownian motion on it. Also we assume that the time horizon $T$ is finite. We denote by $\left(\mathscr{F}_{t}\right)_{0 \leq t \leq T}$ the $P$-completion of the natural filtration of $\left(W_{t}\right), 0 \leq t \leq T$. .

On the filtered probability space $\left(\Omega, \mathscr{F}, \mathscr{F}_{t}, P\right), 0 \leq t \leq T$, we consider a financial market with two assets $\left(S_{t}^{0}, S_{t}, 0 \leq t \leq T\right.$, ) where $S_{t}^{0}$ is the risk-free asset (bank account) and its price at time $t$ is given by

$$
S_{t}^{0}=e^{r t}, \quad 0 \leq t \leq T, r>0, S_{0}^{0}=1,
$$

where as the second asset $S_{t}$ (stock price)is risky and its price at time $t$ is the solution of the following stochastic differential equation

$$
d S_{t}=r S_{t} d t+\eta\left(t, S_{t}\right) S_{t} d W_{t}, \quad 0 \leq t \leq T, S_{0}>0,
$$

where $\eta(t, x):[0, T] \times R_{+} \rightarrow R_{+}$is a positive function and is known as local volatility (function of time and current stock price) and satisfies

$$
0<\underline{\eta} \leq \eta(t, x) \leq \bar{\eta}, \quad 0 \leq t \leq T, x>0 .
$$

We also assume that the diffusion coefficient $x \eta(t, x)$ is globally lipschitz continuous that is

$$
|y \eta(t, y)-x \eta(t, x)| \leq k|y-x|,
$$

\footnotetext{
*Corresponding author e-mail: nasirzainy1@ hotmail.com
} 
where $k$ is a positive constant.

For our need we consider the stochastic differential equation (2) for arbitrary time $u, t \leq u \leq T$ with the initial condition $S_{t}(t, x)=x$ given by

$d S_{u}(t, x)=r S_{u}(t, x) d u+\eta\left(u, S_{u}(t, x)\right) S_{u}(t, x) d W_{u}, \quad t \leq u \leq T$.

It is well known that for the case of American call option its price coincides with the price of the European call option. It means that the American call option should not be exercised before the maturity date $T$. We consider the American put option. Ruling out any arbitrage opportunity let us consider the following pay-off function for the American put option

$$
g(x)=(K-x)^{+}, \quad x \geq 0,
$$

where $K$ is the strike price of the option. It is clear from section 2.7 of Karatzas and Shreve [12], Shreve [14] and Lamberton and Laypere [10] that the price of the American put option is given by

$$
u(t, x)=\sup _{t \leq \tau \leq T} E\left(e^{-r(\tau-t)}\left(K-S_{\tau}(t, x)\right)^{+}\right), \quad 0 \leq t \leq T, x \geq 0,
$$

where $\tau$ denotes the stopping time in the interval $[t, T]$ and the family of stochastic processes $S_{u}(t, x), t \leq u \leq T$ satisfies the stochastic differential equation (5)

\section{Result and Discussion}

In this section we establish our main result about the continuity of the prices of the American put options with the variations in local volatilities. For this we need to consider another local volatility function $\widetilde{\eta}(t, x), 0 \leq t \leq T$ which satisfies inequalities (3), (4) and accordingly the price of the American put option with this volatility is given by

$\widetilde{u}(t, x)=\sup _{t \leq \tau \leq T} E\left(e^{-r(\tau-t)}\left(K-\widetilde{S}_{\tau}(t, x)\right)^{+}\right), \quad 0 \leq t \leq T, x \geq 0$,

where $\tau$ denotes the stopping time in the interval $[t, T]$ but the family of stochastic processes $\widetilde{S}_{u}(t, x), t \leq u \leq T$ satisfies the following stochastic differential equation

$d \widetilde{S}_{u}(t, x)=r \widetilde{S}_{u}(t, x) d u+\widetilde{\eta}\left(u, \widetilde{S}_{u}(t, x)\right) \widetilde{S}_{u}(t, x) d W_{u}, \quad t \leq u \leq T$,

where

$$
\widetilde{S}_{t}(t, x)=S_{t}(t, x)=x .
$$

Achdou [1] has given the same type of estimates for example see Proposition 4.1 there but under the additional regularity assumptions (assumptions 1 and 2 there in). Also he makes use of the Parabolic variational inequalities. Monotonicity in volatility for the American as well as European option prices has been established by Hobson [6] and Ekstrom [3]. Convexity of American option prices has been originally established by Bergman, Grundy and Wiener [2], El-Karoui, Jeanblanc-Picque and Shreve [4] and Hobson [6]. Some estimates for arbitrary finite convex functions are given in Sultan, Pecaric and Shashiashvili [7]. Continuity of the American put option prices has been proved by Rehman and Shashiashvili [13](see sections 2 and 3). Further it has been proved by Sultan and Rehman [8] and Sultan and Shashiashvili [9] that value function is locally lipschitz continuous with respect to the both of its arguments $t$ and $x$. Theorem 2.1 The following relationship is valid for the difference between the American put option prices $u(t, x)$ and $\widetilde{u}(t, x)$ with respect to the corresponding difference of the local volatilities $\eta(t, x)$ and $\widetilde{\eta}(t, x)$ respectively.

$$
|u(t, x)-\widetilde{u}(t, x)| \leq c x \sup _{(t, x)}|\eta(t, x)-\widetilde{\eta}(t, x)|,
$$

where $t \leq u \leq T$ and the constant $c$ depends on $\bar{r}, k$ and $T$.

Proof. We consider the difference of the American put option prices $u(t, x)$ and $\widetilde{u}(t, x)$ given by (7) and (8)

$|u(t, x)-\widetilde{u}(t, x)|=$

$$
\begin{aligned}
& \left|\sup _{t \leq \tau \leq T} E\left(e^{-r(\tau-t)}\left(K-S_{\tau}(t, x)\right)^{+}\right)-\sup _{t \leq \tau \leq T} E\left(e^{-r(\tau-t)}\left(K-\widetilde{S}_{\tau}(t, x)\right)^{+}\right)\right| \\
& \leq \sup _{t \leq \tau \leq T} E\left(e^{-r(\tau-t)}\left|\left(K-S_{\tau}(t, x)\right)^{+}-\left(K-\widetilde{S}_{\tau}(t, x)\right)^{+}\right|\right) \\
& \leq \sup _{t \leq u \leq T} E\left|S_{v}(t, x)-\widetilde{S}_{v}(t, x)\right| \\
& \leq E\left(\sup _{t \leq u \leq T}\left|S_{v}(t, x)-\widetilde{S}_{v}(t, x)\right|\right) .
\end{aligned}
$$

For arbitrary stochastic process $X(\omega)$ we have

$$
E|X(\omega)| \leq\left(E(X(\omega))^{2}\right)^{1 / 2} .
$$

Hence we can write the above inequality in the form

$$
|u(t, x)-\widetilde{u}(t, x)| \leq\left(E\left(\sup _{t \leq u \leq T}\left|S_{u}(t, x)-\widetilde{S}_{u}(t, x)\right|\right)^{2}\right)^{1 / 2} .
$$

Now from equations (4) and (9), it is clear that we can write the stochastic processes $S_{u}(t, x)$ and $\widetilde{S}_{u}(t, x), t \leq u \leq$ $T$ in the forms

$S_{u}(t, x)=\int_{t}^{u} r S_{v}(t, x) d v+\int_{t}^{u} S_{v}(t, x) \eta\left(v, S_{v}(t, x)\right) d W_{v}, \quad t \leq u \leq T$,

and

$\widetilde{S}_{u}(t, x)=\int_{t}^{u} r \widetilde{S}_{v}(t, x) d v+\int_{t}^{u} \widetilde{S}_{v}(t, x) \widetilde{\eta}\left(v, \widetilde{S}_{v}(t, x)\right) d W_{v}, \quad t \leq u \leq T$. 
Consider the difference of the above stochastic processes

$$
\begin{array}{r}
S_{u}(t, x)-\widetilde{S}_{u}(t, x)=\int_{t}^{u} r\left(S_{v}(t, x)-\widetilde{S}_{v}(t, x)\right) d v \\
+\int_{t}^{u}\left(S_{v}(t, x) \eta\left(v, S_{v}(t, x)\right)-\widetilde{S}_{v}(t, x) \widetilde{\eta}\left(v, \widetilde{S}_{v}(t, x)\right)\right) d W_{v} .
\end{array}
$$

Let us denote the difference $S_{u}(t, x)-\widetilde{S}_{u}(t, x)$ by $\widehat{S}_{u}(t, x)$, then we can write the above equation in the form

$$
\widehat{S}_{u}(t, x)=\int_{t}^{u} r \widehat{S}_{v}(t, x) d v+\int_{t}^{u}\left(\widehat{S}_{v}(t, x) \eta+\widetilde{S}_{v}(t, x)(\eta-\widetilde{\eta})\right) d W_{v} .
$$

From here we can write

$$
\begin{gathered}
\sup _{t \leq s \leq u} \widehat{S}_{s}^{2}(t, x) \leq 2 \bar{r}^{2} T \int_{t}^{u} \widehat{S}_{v}^{2}(t, x) d v \\
+2 \sup _{t \leq s \leq u}\left(\int_{t}^{s}\left[\widehat{S}_{v}(t, x) \eta+\widetilde{S}_{v}(t, x)(\eta-\widetilde{\eta})\right] d W_{v}\right)^{2} .
\end{gathered}
$$

Taking mathematical expectation on both sides of the latter inequality together with the use of Doob's classical maximal inequality we get

$$
\begin{gathered}
E \sup _{t \leq s \leq u} \widehat{S}_{s}^{2}(t, x) \leq 2 \bar{r}^{2} T \int_{t}^{u} E \widehat{S}_{v}^{2}(t, x) d v \\
+8 \int_{t}^{u} E\left[\widehat{S}_{v}(t, x) \eta+\widetilde{S}_{v}(t, x)(\eta-\widetilde{\eta})\right]^{2} d v .
\end{gathered}
$$

Let us denote $E \sup _{t \leq s \leq u} \widehat{S}_{s}^{2}(t, x)$ by $\phi(u)$ then from the latter inequality, we obtain $\phi(u) \leq 2 \bar{r}^{2} T \int_{t}^{u} \phi(v) d v+8 \int_{t}^{u} E\left[\widehat{S}_{v}(t, x) \eta+\widetilde{S}_{v}(t, x)(\eta-\widetilde{\eta})\right]^{2} d v$.

Now we bound the last term in the above inequality. We can write

$\widehat{S}_{v} \eta+\widetilde{S}_{v}(\eta-\widetilde{\eta})=S_{v} \eta\left(v, S_{v}\right)-\widetilde{S}_{v} \eta\left(v, \widetilde{S}_{v}\right)+\widetilde{S}_{v}\left(\eta\left(v, \widetilde{S}_{v}\right)-\widetilde{\eta}\left(v, \widetilde{S}_{v}\right)\right)$.

By applying the inequality (4) we can write

$$
\begin{aligned}
\left(S_{v} \eta\left(v, S_{v}\right)-\widetilde{S}_{v} \eta\left(v, \widetilde{S}_{v}\right)\right)^{2} & \leq k^{2}\left(S_{v}-\widetilde{S}_{v}\right)^{2} \\
& =k^{2} \widehat{S}_{v}^{2},
\end{aligned}
$$

and

$$
\left.\left(\widetilde{S}_{v}\left(\eta\left(v, \widetilde{S}_{v}\right)-\widetilde{\eta}\left(v, \widetilde{S}_{v}\right)\right)\right)^{2} \leq \sup _{(t, x)}|\eta(t, x)-\widetilde{\eta}(t, x)|\right)^{2} \widetilde{S}_{v}^{2}
$$

Therefore (18) takes the form

$$
E\left(\widehat{S}_{v} \eta+\widetilde{S}_{v}(\eta-\widetilde{\eta})\right)^{2} \leq 2 k^{2} E \widehat{S}_{v}^{2}+2\left(\sup _{(t, x)}|\eta(t, x)-\widetilde{\eta}(t, x)|\right)^{2} E \widetilde{S}_{v}^{2} .
$$

From Karatzas, Shreve [11](see, for example theorem 2.9, chapter 5) we can bound the term $E \widetilde{S}_{v}^{2}$

$$
E\left(\widetilde{S}_{v}^{2}(t, x) \leq c_{1} x^{2}, \quad t \leq v \leq T,\right.
$$

where $c_{1}$ is a positive constant.

So the previous inequality 20 becomes

$$
E\left(\widehat{S}_{v} \eta+\widetilde{S}_{v}(\eta-\widetilde{\eta})\right)^{2} \leq 2 k^{2} \phi(v)+2 c_{1} x^{2}\left(\sup _{(t, x)}|\eta(t, x)-\widetilde{\eta}(t, x)|\right)^{2} .
$$

Therefore the inequality (17) takes the form

$$
\phi(u) \leq\left(2 \bar{r}^{2} T+16 k^{2}\right) \int_{t}^{u} \phi(v) d v+16 c_{1} x^{2} \int_{t}^{u}\left(\sup _{(t, x)}|\eta(t, x)-\widetilde{\eta}(t, x)|\right)^{2} d v .
$$

Now we apply the classical Gronwall inequality so that the above inequality modifies as

$$
\begin{aligned}
\phi(u) & \leq c_{2} x^{2} \int_{t}^{u}\left(\sup _{(t, x)}|\eta(t, x)-\widetilde{\eta}(t, x)|\right)^{2} d v \\
& \leq c_{2} x^{2} T\left(\sup _{(t, x)}|\eta(t, x)-\widetilde{\eta}(t, x)|\right)^{2}
\end{aligned}
$$

where the constant $c_{2}$ depends on $\bar{r}, k, c_{1}$ and $T$.

From here making the back substitution for $\phi(u)$ and using inequality (12) we come to the conclusion that

$$
|u(t, x)-\widetilde{u}(t, x)| \leq c x \sup _{(t, x)}|\eta(t, x)-\widetilde{\eta}(t, x)|
$$

\section{Conclusion}

We have applied here purely probabilistic approach which enables us to assume only continuity with respect to time for our local volatility functions $\eta(t, x)$ and $\widetilde{\eta}(t, x)$. Also we have given a rigorous proof using this technique. Although it is easy to take the constant volatility as in the case of famous Black-Scholes model but it is now well established that the prices calculated there do not match with the actual market prices see for example Frontczak [5]. So consideration of local volatility is important from this view point. Traders and practitioners dealing in the real-world financial markets use local volatility. So these results may be equally helpful for theorists and practitioners.

\section{References}

[1] Y. Achdou, An inverse problem for a parabolic variational inequality arising in volatility calibration with American options, SIAM J. Control Optim., 43, 1583-1615 (2005).

[2] Y. Z. Bergman, B. D.Grundy, Z. Wiener, General properties of option prices. J. of Finance, 51, 1573-1610 (1996).

[3] E. Ekstrom, Properties of American option prices, Stochastic Process. Appl., 114, 265-278 (2004).

[4] N. El-Karoui, M. Jeanblanc-Picque, S.E. Shreve, Robustness of the Black and Scholes formula, Math. Finance, 8, 93-126 (1998).

[5] R. Frontczak, Valuing options in Heston,s stochastic volatility model: Another analytical approach, J. Appl Math., 2011, (2011). 
[6] D. G. Hobson, Volatility misspecification, option pricing and superreplication via coupling, Ann. Appl. Probab. 8, 193-205 (1998).

[7] S. Hussain, J. Pecaric, M. Shashiashvili, The Weighted Square Integral Inequalities for the First Derivative of the Function of a Real Variable, j. Inequal. Appl., 2008, 343024 (2008).

[8] S. Hussain, N. Rehman, Estimate for the discrete time hedging error of the American option on a dividend paying stock, Math. Inequal. Appl., 15, 137-163 (2012).

[9] S. Hussain, M. Shashiashvili, Discrete time hedging of the American option, Math. Finance. 20, 647-670 (2010) .

[10] P. Jaillet, D. Lamberton, B. Lapeyre, Variational inequalities and the pricing of American options, Acta Appl. Math., 21, 263-289 (1990).

[11] I. Karatzas, S. E. Shreve, Brownian Motion and Stochastic Calculus, 2nd Edn. Springer-Verlag, New York, (1991).

[12] I. Karatzas, S. E. Shreve, Methods of Mathematical Finance, Springer, New York, (1998).

[13] N. Rehman, M. Shashiashvili, The American Foreign Exchange Option in Time-Dependent One-Dimensional Diffusion Model for Exchange Rate, Appl. Math. Optim., 59, 329-363 (2009).

[14] S. E. Shreve, Stochastic Calculus for Finance II, Springer, New York, (2004).

[15] S. Villeneuve, Exercise Regions of American Options on Several Assets, Fin. and Stoch., 3, 295-322 (1999). 